\title{
Are parallaxes of long-period variable stars and red supergiants reliable?
}

\author{
C. Babusiaux ${ }^{1}$ and A. Jorissen ${ }^{2}$ \\ ${ }^{1}$ Observatoire de Paris, Place Jules Janssen, 92195 Meudon, France. \\ email: carine.babusiaux@obspm.fr \\ ${ }^{2}$ IAA, Université Libre de Bruxelles CP 226, 1050 Bruxelles, Belgique. \\ email: ajorisse@astro.ulb.ac.be
}

\begin{abstract}
We have studied the impact of long-term variability and surface brightness asymmetries on the parallaxes of long-period variable stars and red supergiants.
\end{abstract}

Keywords. astrometry, stars: AGB and post-AGB, supergiants

\section{Introduction}

Long-period variables (LPVs) are frequent among highly evolved stars on the asymptotic giant branch (AGB). Period-luminosity relationships have been derived for LPVs in the Large Magellanic Cloud by Wood (2000). It is hoped that Gaia will deliver such relationships for LPVs in the Galaxy (Barthès et al. 1999). The astrometric observations of LPVs and red supergiants face, however, several specific problems that may lower the accuracy of the parallax determination. Their very red colours makes it necessary to apply an adopted chromatic correction. This correction is, however, difficult to calibrate because of the small number of well-behaved stars with such red colours. A good illustration of this difficulty may be obtained from the analysis of the Hipparcos Transit Data (Quist \& Lindegren 1999), which give the parameter fitting of the stellar signal modulated by the grid atop the detector. For a point source, the ratio $R$ of the modulation coefficients of the second over first harmonics should be 0.35 . We observe, however, a clear decrease of this ratio with increasing $V-I$ color. This may explain the large fraction of red stars for which the Hipparcos solution quality flag has been set to S ('suspected non single').

Moreover, the LPVs variability leads to colour variations over the light cycle which calls for the use of epoch colour indices to compute the epoch chromaticity corrections (Platais et al. 2003). Finally, the very large radii of LPVs and supergiant stars make them extended sources and time-varying brightness asymmetries (spots) on their stellar disc could strongly degrade their parallax accuracy (Eriksson \& Lindegren 2007); indeed the angular radius of LPVs can be larger than their parallax as soon as their linear radius exceeds 1 AU.

\section{Hipparcos and the LPVs}

The reprocessing of the Intermediate Astrometric Data (IAD) of LPVs has been made in Brussels using the epoch $V-I$ colour indices (Knapp et al. 2003), leading to much improved parallaxes and also allowed us to reject the Variability-Induced Movers (VIMs) classification for $86 \%$ of the LPVs in the Hipparcos catalogue (Pourbaix et al. 2003). The quality of these parallaxes may be assessed by confronting the parallax standard deviations $\sigma_{\varpi}$ with the $\mathrm{Hp}$ magnitude at minimum brightness (from field $\mathrm{H} 50$ of the Hipparcos Catalogue) instead of with the median Hp. Only a small number of LPVs 


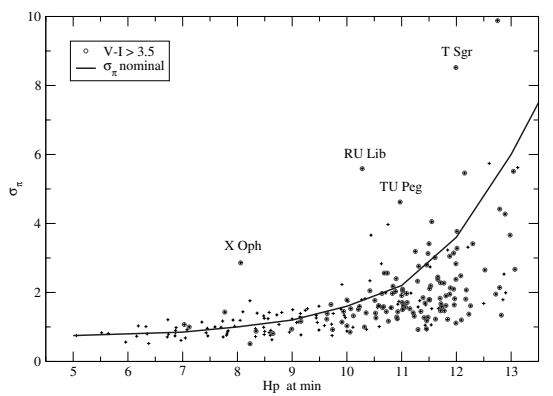

Figure 1. Standard error of the revised parallaxes for LPVs (Knapp et al. 2003) as a function of the Hp magnitude at minimum brightness (95th percentile; field H50 of the Hipparcos catalogue). The solid line is the nominal relation derived from the whole Hipparcos catalogue. Stars redder than $V-I=3.5$ are noted as large dots.

then present too large standard deviations $\sigma_{\varpi}$ with respect to the fiducial relationship $\left(\sigma_{\varpi}, H p\right)$ for the whole Hipparcos Catalogue (see Fig. 3.2.39 of Volume 1), as seen on Fig. 1. At least two of these remaining outliers are known binaries (X Oph, T Sgr). In a forthcoming paper, we show that the goodness-of-fit parameter provides an even more stringent diagnostic than the parallax standard deviation to assess the parallax reliability.

\section{Hipparcos and Betelgeuse}

Supergiants do not show such large variability as LPVs, so that there is no need to re-process their astrometry using epoch colours. Three very bright supergiants show a large excess in their parallax standard error: Betelgeuse, Antares and $\alpha$ Her. The two first have been classified DMSA/X and $\alpha$ Her shows a very poor goodness-of-fit. Interestingly, these three stars are also known to have a surface structure (e.g., Tuthill et al. 1997). During the Hipparcos mission, two interferometric images of the disc of Betelgeuse have been obtained by Wilson et al. (1992) and Tuthill et al. (1997). They reveal a very clear change in the surface brightness which could indeed have affected the Hipparcos measurements. We show in a forthcoming paper that it could explain Betelgeuse's classification as DMSA/X. Depending on the data set used (NDAC, FAST, outliers removal), our reprocessing leads to parallaxes of Betelgeuse between 6.8 and 10.5 mas. The knowledge provided by those interferometric data combined to the new reduction should allow us to provide a better estimate of the Betelgeuse parallax, crucial for the theoretical modelling of this well studied red supergiant.

At the same time, this study provides guidance for designing the reduction pipeline for Gaia in such a way that reduces the possible impact of surface brightness asymmetries on the derived parallaxes.

\section{References}

Barthès, D., Luri, X., Alvarez, R., \& Mennessier, M. O. 1999, A\&SAS 140, 55

Eriksson, U. \& Lindegren, L. 2007, A\& $A$, in press (astro-ph/0706.1646)

Knapp, G. R., Pourbaix, D., Platais, I., \& Jorissen, A. 2003, A $\& A$ 403, 993

Platais, I., Pourbaix, D., Jorissen, A. et al. 2003, $A \& A$ 397, 997

Pourbaix, D., Platais, I., Detournay, S. et al. 2003, A\&A 399, 1167

Quist C. F. \& Lindegren L. 1999, A\&SAS 138, 327

Tuthill, P. G., Haniff, C. A., \& Baldwin, J. E. 1997, MNRAS 285, 529

Wilson, R. W., Baldwin, J. E., Buscher, D. F., \& Warner, P. J. 1992, MNRAS 257, 369

Wood P. R. 2000, PASA 17, 18 